\title{
Desenvolvimento de um Laboratório Remoto de FPGA: Indo em Direção a um Laboratório Híbrido
}

João Pedro Ballerini Bruno, Unifesp,jpedrobruno@ hotmail.com, https://orcid.org/00000002-8858-6864

Tiago Oliveira, Unifesp,tiago.oliveira@unifesp.br, https://orcid.org/0000-0002-3676$\underline{5967}$

André Marcorin de Oliveira, Unifesp,andre.marcorin@ unifesp.br,https://orcid.org/00000003-4351-3896

Resumo: Este artigo descreve o desenvolvimento de um laboratório remoto de FPGA para o aprendizado de circuitos digitais. A solução proposta (laboratório remoto) faz uso da aplicação VirtualHere e se integra ao software WiRED Panda, o qual é um simulador de circuitos de lógica digital (laboratório virtual). A solução proposta foi implantada no setor de Tecnologia de Informação de uma universidade e validada por meio de testes e questionários com os alunos participantes da pesquisa, obtendo-se resultados promissores quanto a sua utilização.

Palavras-chave: laboratório remoto FPGA, laboratório híbrido, projeto de circuitos digitais.

\section{Development of an FPGA Remote Laboratory: Towards a Hybrid Laboratory}

Abstract: This article describes the project of an FPGA remote laboratory for digital circuit learning. The proposed solution (remote laboratory) is supported by VirtualHere application, and it is integrated with WiRED Panda software that can be seen as a digital circuit simulator (virtual laboratory). The proposed solution was implemented at Information Technology department of a university, and it was validated by means of tests and questionnaire applied with students that participated of the research. The analysis of the research has demonstrated promising results towards its use.

Keywords: FPGA remote laboratory, hybrid laboratory, digital circuit design.

\section{Introdução e Contextualização}

Ao avançar dos anos, a revolução digital continua a transformar a vida das pessoas, trazendo diversas mudanças de hábitos para toda a população. Dentre essas transformações, podemos citar a transformação do mercado de trabalho, a inclusão gerada pela facilidade na realização de conferências remotas, as mudanças no ensino superior que contam agora com um público maior e um conteúdo técnico cada vez mais extenso (Raschke, 2003). Devido as mudanças nos hábitos, muitas são as tarefas que hoje são realizadas por meio de um sistema computacional.

Focando-se no escopo educacional, o acesso à informação tem sido facilitado, permitindo que o aluno se torne menos dependente e passe a ser cada vez mais protagonista de seu aprendizado, de forma que os professores tenham suas funções voltadas ao auxílio e direcionamento de conteúdos programáticos. Disciplinas predominantemente teóricas podem então ser compreendidas sem a necessidade de interações presenciais, de forma que o acompanhamento do aprendizado possa ser realizado por meio de trabalhos, questionários, apresentações e outras formas de acompanhamento. Por sua vez, as disciplinas predominantemente práticas podem impor 
uma dificuldade adicional, se não forem utilizadas ou desenvolvidas as devidas ferramentas necessárias.

De modo a suprir esta limitação, universidades têm optado por desenvolver laboratórios que possam ser manuseados de forma remota, sendo assim possível observar os fenômenos empíricos mesmo sem a presença do aluno (Álvares e Ferreira, 2003).

Neste contexto, durante o processo de graduação nos cursos de Engenharia da Computação, Elétrica e Ciência da Computação, avalia-se a vasta presença das unidades curriculares voltadas a compreensão e prática de sistemas e circuitos digitais dada as diretrizes curriculares nacionais (MEC, 2016). Especificamente no contexto da Universidade Federal de São Paulo (Unifesp), os alunos dos cursos voltados à área da computação fazem uso do laboratório de Hardware e Redes de Computadores, onde utilizam em grande parte de suas formações um kit de desenvolvimento, com o intuito de implementar circuitos de sistemas computacionais projetados pelos alunos no decorrer do curso. Este kit conta com a presença de um Field Programmable Gate Array (FPGA), um dispositivo lógico programável (Braggio, 2014). Esses dispositivos são utilizados em pelo menos cinco unidades curriculares e devido ao alto custo do equipamento, sua aquisição pelos alunos da universidade é impraticável, restringindo o desenvolvimento das tarefas ao horário das disciplinas. Dada a complexidade dos projetos que avançam no decorrer do curso, esta restrição torna-se um fator limitante ao aprendizado.

Tendo sido contextualizado o problema, o objetivo geral do trabalho é a implementação de um laboratório remoto de FPGA utilizando como base a aplicação WiRED Panda de modo a permitir a realização de conexões remotas com dispositivos como o kit de desenvolvimento FPGA DE2-115.

$\mathrm{Na}$ próxima seção, encontra-se uma breve fundamentação teórica sobre Laboratórios Remotos, Virtuais e Híbridos. Por sua vez, na seção 3 encontram-se os materiais e métodos utilizados na construção do laboratório remoto de FPGA proposto, bem como se apresenta a metodologia de avaliação do laboratório desenvolvido. Na seção 4, discorre-se sobre os resultados obtidos com os testes realizados e, na seção 5, apresentase a conclusão desse artigo.

\section{Laboratórios Remotos, Virtuais e Híbridos}

Por definição, laboratório remoto é aquele que pode ser utilizado de forma totalmente remota, sendo possível alterar parâmetros de entrada e analisar respostas empíricas, passando uma experiência similar a estar presente em um laboratório prático (Lourenço, 2014).

Desde 2001, foi constatado que o uso de laboratórios remotos enaltece a vontade dos alunos de se aprofundarem cada vez mais na disciplina (Lindsay et al., 2007). A capacidade de realizar os experimentos sem a necessidade de estar presente no laboratório instiga os alunos a realizar as experimentações em momentos extra-classe, assim como possibilita a revisão e a realização de experimentos, mesmo em caso de ausência em aulas presenciais.

Também se discute quanto a real necessidade de um laboratório remoto, uma vez que existe a possibilidade do uso de simuladores ou laboratórios virtuais, os quais visam imitar os experimentos avaliados na realidade. Os laboratórios virtuais prometem ser menos limitados e mais amigáveis para o aprendizado. No entanto, embora soluções virtuais contribuam positivamente para o aprendizado, estas muitas vezes são insuficientes para simular o comportamento e as dificuldades encontradas na realização de experimentos em bancada, o que poderia contribuir para o empobrecimento do conhecimento prático (Vidal e Menezes, 2015). 
Por sua vez, como o próprio termo híbrido já enuncia, os laboratórios híbridos são aqueles que possuem tanto aspectos remotos quanto virtuais, combinando, portanto, componentes simulados (termo virtual) e equipamentos reais (termo remoto) (RODRIGUEZ-GIL et. al, 2016). Esses laboratórios prometem trazer um pouco dos dois mundos colocando a atividade prática nas mãos do aluno e o direcionando para aplicações em diferentes cenários. Neste contexto, o ideal é que um laboratório híbrido possa permitir interação e integração entre componentes reais e componentes simulados de forma transparente e facilitada aos seus usuários.

O trabalho de (RODRIGUEZ-GIL et. al, 2016) explora uma solução web de laboratório híbrido de FPGA, trazendo um sistema específico que integra um FPGA com componentes virtuais de modelo industrial visando o controle de uma caixa d'água. Uma vantagem de se trabalhar com soluções virtuais e remotas refere-se à possibilidade de se incluir outras funcionalidades computacionais ao sistema, como por exemplo, no trabalho apresentado em (ANDÚJAR et. al, 2011), que traz recursos de realidade aumentada em um laboratório remoto.

\section{Metodologia de Condução e Desenvolvimento do Projeto}

Para a condução desse trabalho de pesquisa, primeiramente foi projetado o laboratório remoto de FPGA (seção 3.1), instalando-o no setor de Tecnologia da Informação da Unifesp, e por fim foram realizados dois testes sobre o projeto desenvolvido, sendo eles: teste de responsividade e teste de usabilidade (seção 3.2). Ao todo, 8 alunos do curso de Bacharelado em Ciência e Tecnologia e Bacharelado em Engenharia de Computação da Unifesp participaram do teste do sistema projetado. A possibilidade de participação no teste foi divulgada por email e redes sociais, devendo o aluno ter conhecimento de Circuitos Digitais ou Arquitetura e Organização de Computadores.

\subsection{Materiais e Métodos}

Para o desenvolvimento do laboratório remoto de FPGA foram utilizados alguns softwares e equipamentos específicos que interligados permitiram a implementação da solução e o acesso remoto do kit FPGA pelos estudantes.

Em relação aos principais softwares utilizados, pode-se citar: WiRED Panda e VirtualHere. O WiRED Panda é um software livre licenciado sob a licença GPL-3.0 e o código fonte está disponível no GitHub, plataforma online de versionamento e consulta de códigos. O WiRED Panda foi desenvolvido na Unifesp para ser um simulador de circuitos de lógica digital intuitivo e fácil de operar, sendo utilizado em ambientes de aprendizado de disciplinas relacionadas a Circuitos Digitais e Arquitetura e Organização de Computadores.

Por sua vez, com o propósito de empregar dispositivos periféricos USB de forma remota, a aplicação comercial VirtualHere oferece um meio de realizar a conexão dos dispositivos através de uma rede, sendo ideal para evitar que estes precisem estar conectados nos computadores em que são utilizados. De forma transparente ao usuário, o VirtualHere oferece drivers disponíveis para os sistemas Windows, Linux e MacOS que virtualmente conectam dispositivos no computador do cliente sem que exista a necessidade destes serem feitos para operar de forma remota. Como o processo é feito a nível de protocolo, este é transparente aos drivers dos próprios dispositivos. Caso a velocidade de conexão não seja um pré-requisito, qualquer periférico USB que funcionar nos sistemas operacionais poderão ser utilizados de forma remota, mesmo estando conectado em outro computador. 
Outros trabalhos que fizeram uso do VirtualHere relataram apresentar um bom desempenho, mesmo quando utilizados em redes internet. Um exemplo de aplicação do VirtualHere no Brasil foi na Universidade Federal de Uberlândia, onde desenvolveu-se um laboratório de baixo custo utilizando computadores thin-clients que atuam apenas como terminais controlados por um servidor de processamento central (Silva et al., 2019). A solução possibilitou a integração dos controladores do laboratório nos terminais conforme o uso. Em (Pintilie et al., 2018) foi redigido um trabalho que utilizou esta solução para conectar dispositivos periféricos através da rede elétrica. Para isto, foram utilizados adaptadores para criar uma rede ethernet através da geração de ruídos de alta frequência na instalação elétrica de modo que possibilitou o desenvolvimento de um socket de tomada multi-função.

Em relação aos principais equipamentos, na Tabela 1 foram inseridos todos os produtos que podem ser utilizados na realização do laboratório remoto de FPGA. Contudo há mais de uma opção de um mesmo produto, como é o exemplo do Raspberry Pi. O custo foi calculado levando em consideração a compra recomendada dos componentes e a compra mínima - é importante ressaltar que itens como fios, jumpers, protoboard e relês não estão incluídos na aquisição mínima, devido ao baixo custo destes itens e ao fato de estarem disponíveis em praticamente todas as lojas de produtos eletrônicos.

Tabela 1 - Componentes necessários para a montagem do Laboratório Remoto.

\begin{tabular}{|l|l|l|c|c|}
\hline \multicolumn{1}{|c|}{ Produto } & \multicolumn{1}{|c|}{ Loja } & $\begin{array}{c}\text { Preço } \\
\text { U\$ }\end{array}$ & Recomendado & Mínimo \\
\hline Raspberry Pi 4 4GB & Amazon (EUA) & 61,88 & $\mathrm{X}$ & \\
\hline Raspberry Pi 4 2GB & Amazon (EUA) & 52,99 & & $\mathrm{X}$ \\
\hline RPI4 - Case, Heatsinks and Fan & Amazon (EUA) & 10,99 & $\mathrm{X}$ & \\
\hline RPI4 - Power SUpply & Amazon (EUA) & 9,99 & $\mathrm{X}$ & $\mathrm{X}$ \\
\hline MicroSDHC - 32GB & Amazon (EUA) & 7,49 & $\mathrm{X}$ & $\mathrm{X}$ \\
\hline Arduíno Board ATmega 2560 & Amazon (EUA) & 36,28 & $\mathrm{X}$ & \\
\hline ATmega 2560 - Generic & Amazon (EUA) & 17,99 & & $\mathrm{X}$ \\
\hline ELEGOO 830pts Breadboard (3pcs) & Amazon (EUA) & 9,99 & $\mathrm{X}$ & \\
\hline ELEGOO Jumper Wires (120 pcs) & Amazon (EUA) & 7,65 & $\mathrm{X}$ & \\
\hline Arduíno Relay Switch 5V (2pcs) & Amazon (EUA) & 5,79 & $\mathrm{X}$ & \\
\hline DEV KIT DE2-115 Cyclone IV & Mouser Elec. & 595,00 & & \\
\hline DEV KIT DE0-Nano Cyclone IV & Amazon (EUA) & 121,71 & & $\mathrm{X}$ \\
\hline DEV KIT DE0-Nano Cyclone IV & TerasIC & 79,00 & & $\mathrm{X}$ \\
\hline DEV KIT DE0-Nano-SoC Cyclone V & TerasIC & 99,00 & $\mathrm{X}$ & \\
\hline Licença VirtualHere & VirtualHere Pty & 49,00 & $\mathrm{X}$ & $\mathrm{X}$ \\
\hline
\end{tabular}

Diversos kits de desenvolvimento com FPGA foram inseridos na Tabela 1, visto que seu uso pode variar de acordo com o requisito do projeto. A instalação realizada neste trabalho fez uso do kit FPGA DE2-115, pois este já estava disponível na Universidade. Contudo não é interessante adquiri-los para este propósito, pois este possui muito mais funcionalidades do que será possível utilizar. Deste modo, foram incluídas outras opções de compra que estão mais alinhadas com o intuito do projeto. Neste caso o kit FPGA DE0-Nano da Altera é mais indicado, pois possui uma maior quantidade de pinos acessíveis que poderão ser mapeados para o uso remoto. O kit FGA DE0-Nano possui duas versões. A versão mais nova possui um componente FPGA Cyclone $\mathrm{V}$ e um processador Cortex-A9 de arquitetura ARM integrado. Embora esta versão seja um pouco mais cara, ela irá ampliar as possibilidades de uso do equipamento.

Foi utilizado no projeto um ATmega 2560 genérico. Devido a isso, alguns problemas de compatibilidade foram encontrados sendo necessário utilizar drivers diferenciados para funcionar. Para prevenção de eventuais incompatibilidades é sugerido o kit original (italiano). 
Em relação a licença para uso do VirtualHere, embora tenha o preço equivalente a um Raspberry PI, seu uso é perpétuo, ou seja, não há a necessidade de renová-la. Entretanto, vale salientar que no caso do servidor deixar de funcionar e for necessário mudanças no hardware, estas poderão invalidar a licença.

Quanto ao Raspberry PI, não há motivos para selecionar a versão de 4GB ao invés da versão de $2 \mathrm{~GB}$, isto porque o laboratório remoto desenvolvido nesse projeto usa menos de $768 \mathrm{MB}$ de memória principal. Contudo, vale lembrar que ao fazer a aquisição da licença VirtualHere, será necessário associar a licença a um dispositivo e, como a licença é intransferível, é interessante optar por uma versão superior para que não tenha que fazer uma nova aquisição em caso de necessidade. Por este mesmo motivo, não é recomendado fazer uso de um Raspberry PI 3B ou anterior, embora, ao menos para o VirtualHere um Raspberry PI 3B seja suficiente.

Utilizando os softwares e equipamentos comentados nos parágrafos anteriores, a arquitetura do laboratório remoto de FPGA desenvolvido é composta de quatro partes principais, a aplicação WiRED Panda instalada na máquina do usuário, o serviço VirtualHere com as suas customizações e integrações com o sistema, assim como o serviço responsável pela autenticação dos usuários e comunicação entre a aplicação cliente e o controlador programável que efetivamente conectará o kit de desenvolvimento ao serviço (Figura 1). A arquitetura da solução proposta encontra-se na Figura 1.

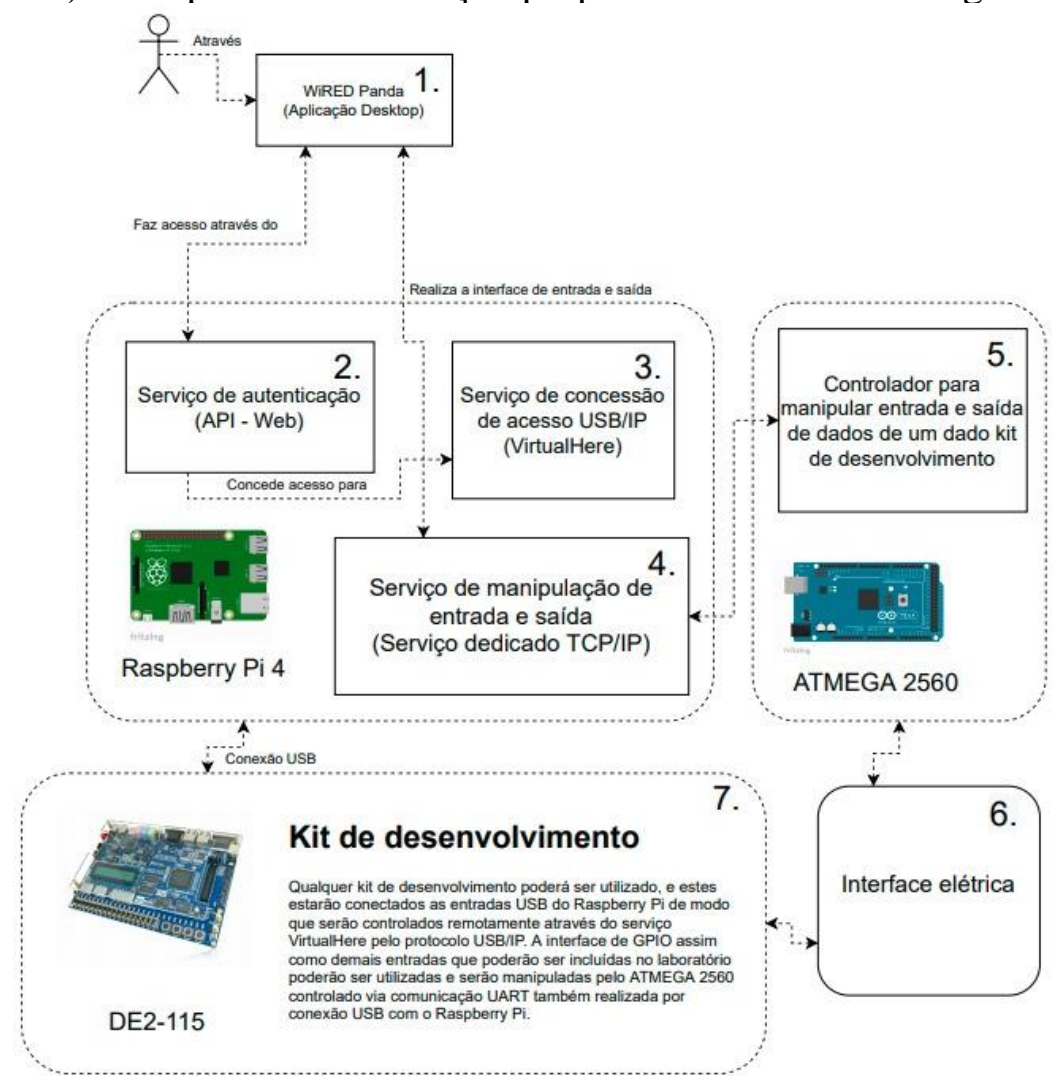

Figura 1 - Arquitetura da solução proposta.

Tendo sido implantada a solução proposta no setor de Tecnologia da Informação na Unifesp, uma exemplificação de uso do laboratório remoto encontra-se na Figura 2. Nessa figura, apresenta-se um exemplo de um contador, sendo que toda vez que for pressionado com o mouse o botão "next", será mostrado no display LCD o próximo número da sequência decimal. A figura 2 traz o WiRED Panda como ambiente de trabalho, sendo que o componente "CONF REMOTE" refere-se à instanciação no WiRED Panda do laboratório remoto proposto. 


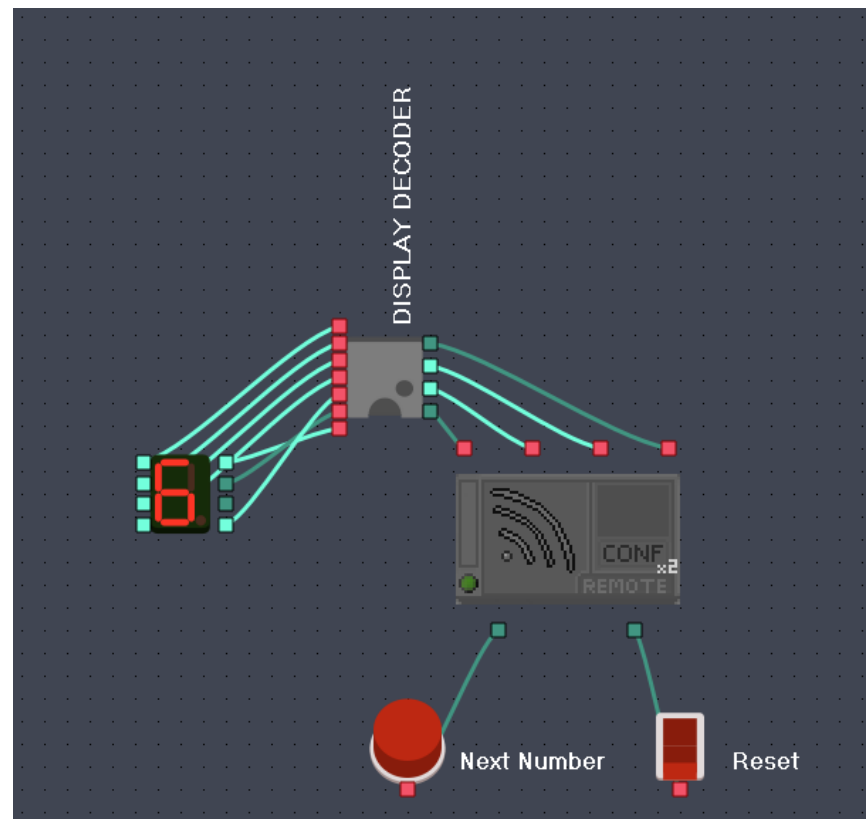

Figura 2 - Exemplo de um circuito contador no WiRED Panda com o instanciamento do Laboratório Remoto.

\subsection{Avaliação do Laboratório Remoto Desenvolvido}

De modo a avaliarmos como tem sido a aceitação dos alunos com a solução do laboratório remoto, realizou-se um questionário com os alunos após suas primeiras interações com o sistema. Esta avaliação está baseada no System Usability Scale (SUS). Este é um padrão de questionário para avaliar a usabilidade de algum sistema ou equipamento, tendo sido inicialmente apresentado em 1996 por John Brooke (Brooke, 1996), e sua publicação original possui 10381 citações, segundo a plataforma Scholar Google (avaliado no dia 11/02/2021). Este padrão de questionário foi utilizado em 2009 por aproximadamente $40 \%$ das indústrias que realizavam avaliações de usabilidade segundo Sauro e Lewis (2009).

Os pontos explorados por estas questões avaliam os seguintes critérios:

- Capacidade de aprendizagem e o quão fácil é utilizá-lo pela primeira vez;

- Rapidez com que os resultados esperados são entregues, seja em facilidade de realizar as tarefas, ou em responsividade;

- Intuitividade, sendo assim fácil de memorizar as instruções necessárias para operá-lo;

- Ausência de comportamentos inesperados, seja devido a erros ou relacionado a baixa Quality of Service (QoS);

- Satisfação gerada pelo uso do sistema, em termos de design ou quanto dinamicidade das operações.

Alguns destes critérios foram descritos por Jacob Nielsen (2003) e compõem os fatores que levam o sistema a ter baixo ou alto fator de usabilidade, sendo explorado pelo SUS as questões a serem elencadas para considerar estes fatores.

Além de prover perguntas formuladas para avaliar os diversos pontos relevantes da usabilidade, o SUS também é capaz de gerar uma pontuação final com base nas respostas, pontuação esta avaliada no intervalo de 0 a 100 que deverá classificar o sistema analisado. Em um trabalho realizado em 2009 (Bangor et al., 2009), foram relacionadas 
as pontuações SUS com adjetivos que ajudam a melhor compreender o significado destas pontuações, onde foi possível extrair a Tabela 2.

Tabela 2 - Classificação do sistema avaliado em relação à pontuação SUS.

\begin{tabular}{|l|l|l|l|}
\hline \multicolumn{1}{|c|}{ Adjetivo } & \multicolumn{1}{c|}{ Pontuação } & \multicolumn{1}{c|}{ Média Score SUS } & \multicolumn{1}{c|}{ Desvio Padrão } \\
\hline Pior imaginável & 4 & 12,5 & 13,1 \\
\hline Péssimo & 22 & 20,3 & 11,3 \\
\hline Ruim & 72 & 35,7 & 12,6 \\
\hline OK & 211 & 50,9 & 13,8 \\
\hline Bom & 345 & 71,4 & 11,6 \\
\hline Excelente & 289 & 85,5 & 10,4 \\
\hline Melhor imaginável & 16 & 90,9 & 13,4 \\
\hline
\end{tabular}

Além do questionário SUS, outras seis perguntas também foram adicionadas para fazer uma análise quanto ao contexto em que o aluno fez uso do sistema, sua prospecção quanto ao momento em que este laboratório se tornou disponível e se o aluno foi capaz de utilizá-lo, visando avaliar a compatibilidade da solução com conexões de alta latência ou instáveis. Segue a lista das perguntas realizadas:

1. Fui capaz de utilizar o laboratório remoto;

2. Acredito que gostaria de utilizar este sistema frequentemente*;

3. Achei o sistema desnecessariamente complexo*;

4. Achei o sistema fácil de usar*;

5. Acredito que precisaria do apoio de um suporte técnico para fazer uso do sistema*;

6. Achei que as funções presentes neste sistema foram bem integradas*;

7. Achei que houve muitas inconsistências neste sistema*;

8. Imagino que a maioria das pessoas aprenderiam a usar esse sistema rapidamente*;

9. Achei o sistema muito pesado para utilizá-lo*;

10. Me senti bastante confiante usando esse sistema*;

11. Houve a necessidade de aprender uma série de coisas antes que eu pudesse continuar a utilizar esse sistema*;

12. Tive contato com a ferramenta WiRED Panda anteriormente;

13. Acredito que o momento de disponibilização desta ferramenta é oportuno;

14. Acredito que esta ferramenta torna as possibilidades de desenvolvimento de circuitos menos limitadas, contribuindo positivamente para o desenvolvimento das atividades;

15. Qual foi a tecnologia de rede utilizada para conectar-se a internet durante o uso da ferramenta;

16. Qual dos laboratórios você se encontra em sua trajetória acadêmica.

As questões indicadas com um asterisco são as questões do SUS e foram avaliadas por meio da escala Likert assinalada pelo respondente. Estas questões foram somente direcionadas ao aluno que respondeu positivamente a primeira questão. A questão 12 foi respondida de forma lógica (Booleana), podendo influenciar na pontuação do SUS. Já as questões 13 e 14, foram respondidas por meio da escala Likert e foram utilizadas para traçar paralelos ao contexto. Nas duas últimas questões, foram dadas diferentes opções de escolha para avaliar o impacto da qualidade da conexão do aluno na pontuação de usabilidade e também avaliar o contexto dos alunos que estão fazendo uso desta solução.

\section{Resultados e Discussões}

Após instalado o laboratório remoto no setor de Tecnologia da Informação na Unifesp, foram realizados alguns testes para classificar a latência fazendo uso do sistema de redirecionamento com serviço em nuvem (cloud), e também através da rede privativa 
(VPN) da Universidade. Além dos testes para avaliar o tempo de responsividade da solução, durante o período de uma semana, os estudantes fizeram uso da solução e responderam ao questionário comentado na subseção 3.2. O laboratório remoto instalado contou com um kit FPGA, o que permitiu a utilização de um aluno por vez (para acesso simultâneo há a necessidade de um kit por aluno).

\subsection{Testes de responsividade da solução}

Utilizando o laboratório remoto implantado, foi avaliada a latência de diferentes cidades, com diferentes formas de conexão, utilizando ou não o acesso externo (que faz uso do serviço de redirecionamento de pacotes). Os resultados foram inseridos na Tabela 3.

Tabela 3 - Responsividade do Laboratório Remoto.

\begin{tabular}{|l|c|c|}
\hline \multirow{2}{*}{\multicolumn{1}{c|}{ Localização }} & \multicolumn{2}{|c|}{ Latência Média (ms) } \\
\cline { 2 - 3 } & Externo & Interno \\
\hline Interior de São Paulo & 15 & 13 \\
\hline São José dos Campos & 14 & 11 \\
\hline São Paulo & 8 & 8 \\
\hline
\end{tabular}

Alguns pontos a serem considerados:

- A medição avaliada como "Interior de São Paulo" foi realizada com dados da cidade de Campinas e São João da Boa Vista.

- A latência foi medida através da própria ferramenta WiRED Panda, calculando a diferença do tempo do envio de uma mensagem e sua respectiva resposta.

- Todas as medições foram realizadas utilizando provedores de internet com tecnologia Fiber to the home (FTTH).

Estes resultados são importantes devido a existência de uma latência máxima recomendada para uso da solução VirtualHere, esta, segundo o desenvolvedor, é de $35 \mathrm{~ms}$. Com os resultados obtidos, é possível garantir o funcionamento deste método de conexão com uma boa margem para conexões FTTH.

Também foram realizados testes utilizando conexão móvel de tecnologia 4G, tendo sido possível obter uma latência média de $50 \mathrm{~ms}$. Mesmo apresentando uma latência superior a recomendada (de $35 \mathrm{~ms}$ ), foi possível fazer uso da solução normalmente.

Testes com conexão móvel de tecnologia $3 \mathrm{G}$ também foram realizados, contudo devido à alta instabilidade e grandes variações na latência, não foi possível fazer uso da solução, abrindo precedentes para trabalhos futuros que envolvam a integração com outro método de conexão para atender aos alunos com conexões de alta instabilidade.

Apenas como critério comparativo, a velocidade com que um pacote é enviado e retornado na pior medição $(15 \mathrm{~ms})$, foi superior a velocidade de atualização da imagem de um monitor convencional (que opera a uma taxa de $60 \mathrm{~Hz}$ ), o que permite conferir uma alta responsividade do sistema.

Vale a pena ressaltar que embora a latência medida seja extremamente baixa, o tempo para atualizar uma saída de um determinado circuito digital que está sendo mapeado no laboratório remoto de FPGA deve ser adicionado ao tempo necessário para o microcontrolador fazer a alteração do valor de entrada, e notificar a mudança na saída para o Raspberry PI. 


\subsection{Resultado do questionário}

Durante os sete dias de pesquisa foram obtidos um total de 8 avaliações. A solução foi pontuada pela pontuação SUS com nota 94,69, algo que se encaixaria no adjetivo Melhor imaginável (Bangor et al., 2009). Segundo Nunnally (1978), é necessário ao menos a participação de cinco pessoas para assegurar uma estimativa estável da pontuação. Entretanto, quanto maior o número de avaliações, maior será a confiança atrelada a esta pontuação. As respostas obtidas encontram-se na Tabela 4.

Tabela 4 - Respostas para as perguntas do SUS.

\begin{tabular}{|l|l|c|c|c|c|c|}
\hline \multirow{2}{*}{ Id } & \multicolumn{1}{|c|}{ Perguntas } & \multicolumn{5}{c|}{ Respostas } \\
\cline { 3 - 7 } & & $\mathbf{1}$ & $\mathbf{2}$ & $\mathbf{3}$ & $\mathbf{4}$ & $\mathbf{5}$ \\
\hline 1. & Acredito que gostaria de utilizar este sistema frequentemente. & 1 & 0 & 1 & 1 & 5 \\
\hline 2. & Achei o sistema desnecessariamente complexo. & 7 & 1 & 0 & 0 & 0 \\
\hline 3. & Achei o sistema fácil de usar. & 0 & 0 & 1 & 1 & 6 \\
\hline 4. & $\begin{array}{l}\text { Acredito que precisaria do apoio de um suporte técnico para } \\
\text { fazer uso do sistema. }\end{array}$ & 7 & 0 & 1 & 0 & 0 \\
\hline 5. & $\begin{array}{l}\text { Achei que as funções presentes nesse sistema foram bem } \\
\text { integradas. }\end{array}$ & 0 & 0 & 0 & 0 & 8 \\
\hline 6. & Achei que houve muitas inconsistências nesse sistema. & 8 & 0 & 0 & 0 & 0 \\
\hline 7. & $\begin{array}{l}\text { Imagino que a maioria das pessoas aprenderiam a usar esse } \\
\text { sistema rapidamente. }\end{array}$ & 0 & 0 & 0 & 2 & 6 \\
\hline 8. & Achei o sistema muito pesado para utilizá-lo. & 7 & 1 & 0 & 0 & 0 \\
\hline 9. & Me senti bastante confiante usando esse sistema. & 0 & 0 & 0 & 1 & 7 \\
\hline 10. & $\begin{array}{l}\text { Houve a necessidade de aprender uma série de coisas antes } \\
\text { que eu pudesse continuar a utilizar esse sistema. }\end{array}$ & 8 & 0 & 0 & 0 & 0 \\
\hline
\end{tabular}

Além das perguntas referentes ao questionário SUS, algumas outras perguntas foram adicionadas como descritas na subseção 3.2. Foi realizado também um levantamento quanto ao uso prévio do simulador WiRED Panda, isto devido ao fato de que alunos que já fizeram uso do simulador estão acostumados a utilizá-lo, podendo impactar no questionário de usabilidade do laboratório remoto utilizado.

Ao avaliarmos a pontuação SUS apenas daqueles que tinham tido contato anteriormente com o software WiRED Panda, obtemos uma pontuação de 93,75. Embora tenham sido realizadas poucas avaliações, esta análise poderia sugerir que a própria usabilidade do software WiRED Panda tenha tido impacto na pesquisa, isto porque os alunos que utilizavam previamente a ferramenta, por já estarem acostumados a fazer uso desta, podem ter focado a avaliação do laboratório remoto ao invés de terem avaliado todo o conjunto (laboratório remoto + software WiRED Panda).

Dentre aos comentários realizados pelos estudantes, há duas sugestões para melhorar a usabilidade do sistema, sendo uma delas a correção de um problema que fazia a janela de autenticação fechar após iniciar a conexão ou ser direcionado a fila - fazendo necessário ao usuário abrir novamente o menu de configurações. A outra sugestão foi referente à parte de realização da pinagem, que no caso, não aceita o clique duplo como forma de seleção rápida do pino da lista, fazendo com que o usuário tenha que selecionar o pino e em seguida pressionar o botão Ok.

Outras sugestões apresentadas pelos alunos foram: aumentar a quantidade de pinos de entrada/saída para utilização e adicionar o componente do display LCD no projeto.

É possível fazer o aumento da quantidade de pinos ao substituir um maior número de chaves físicas do kit de desenvolvimento DE2-115, ou fazendo uso de um outro kit de 
desenvolvimento com uma maior quantidade de pinos disponíveis para propósito geral. Entretanto deve-se avaliar que o microcontrolador utilizado no momento é um ATmega 2560, e este embora apresente uma quantidade grande de pinos, também é um fator limitante (máximo de 54 pinos digitais e 16 pinos analógicos).

Quanto ao display LCD, é possível adiciona-lo ao simulador visto que sua operação é assíncrona, sendo compatível com esta solução. Entretanto, será necessário configurar o dispositivo para que o pulso do Enable dure um tempo maior, e que tenha duração suficiente para ser reconhecido pelo microcontrolador e repassado para o usuário. A implementação, porém, não é trivial, devido aos inúmeros comandos a serem implementados e funcionalidades que esse componente físico possui.

Para as duas perguntas na escala Likert incluídas (13 - Acredito que o momento de disponibilização desta ferramenta é oportuno e 14 - Acredito que esta ferramenta torna as possibilidades de desenvolvimento de circuitos digitais menos limitadas, contribuindo positivamente para o desenvolvimento das atividades), foram obtidas as seguintes médias aritméticas: 4,55 para a questão 13 e 4,77 para a questão 14. A primeira delas avalia a compatibilidade do projeto com o contexto sendo vivenciado. O alto índice de respostas positivas pode estar relacionado com o fato de o ensino estar sendo realizado apenas de forma remota, devido ao contexto atual da pandemia, que tem exigido distanciamento social e a realização de aulas não presenciais. O outro ponto avaliado refere-se a perspectiva dos alunos sobre as possibilidades que esta ferramenta pode trazer para a execução das atividades. Avaliado na escala Likert, apenas um aluno foi indiferente, sendo que os demais pontuaram com "Concordo totalmente". A ideia geral desta pergunta foi avaliar a compreensão dos alunos quanto a praticidade do laboratório remoto, podendo concluir que os alunos perceberam que tornando os dispositivos de entrada e saída desacoplados do kit de desenvolvimento, estes podem fazer uso de componentes antes não disponíveis para uso. Além disso, os alunos perceberam que podem distribuir os pinos entre diferentes componentes conforme a necessidade, não sendo limitado a utilizar somente os recursos presentes no kit.

\section{Considerações Finais}

No presente trabalho foi possível desenvolver um método para a utilização do kit de desenvolvimento FPGA DE2-115 de forma remota. A solução foi desenvolvida de forma genérica e acoplada ao simulador de circuitos digitais WiRED Panda, sendo escalável também para ser utilizada com outros kits de desenvolvimento FPGA.

Fazendo uso do software VirtualHere de forma integrada a solução, foi possível aproximar o uso do laboratório remoto ao uso presencial, não limitando o aluno a funcionalidade única de realizar a reprogramação.

Após desenvolvida e implantada no setor de Tecnologia de Informação da Unifesp, a solução foi disponibilizada para os alunos realizarem testes e validarem o seu funcionamento, tendo sido obtido resultados bastante positivos quanto a sua utilização.

Além disso, vale aqui ressaltar que o laboratório remoto desenvolvido se integra ao software WiRED Panda, o qual por sua vez é um simulador de experimentos de circuitos digitais (laboratório virtual). Sendo assim, a plataforma final (simulador WiRED Panda + laboratório remoto desenvolvido) se classificaria como um laboratório híbrido, como comentado na seção 2 , trazendo as funcionalidades de um simulador integrado a um laboratório remoto.

As principais vantagens do resultado final ter sido um laboratório híbrido se dá justamente na possibilidade do aluno poder projetar parte da lógica no simulador, e a outra 
parte no componente controlado remotamente, como demonstrado no exemplo da Figura 2, sendo parte desempenhada pelo componente remoto FPGA e a outra parte simulada.

Por fim, vale comentar que na revisão sistemática da literatura publicada em (Beraldo et al., 2020), foi observado que as pesquisas sobre laboratórios remotos exclusivamente de FPGAs não são em grande número e estão sendo realizadas em países estrangeiros. Dentro desse contexto, deve-se ressaltar que o laboratório remoto de FPGA foi desenvolvido nacionalmente, bem como o software com o qual ele se integra, o WiRED Panda. Com soluções nacionais, pode-se aplicá-los em ambientes educacionais brasileiros, visando atender demandas mais contextualizadas e específicas.

\section{Referências}

ÁLVARES, A. J.; FERREIRA, J. C. E. Metodologia para implantação de laboratórios remotos via internet na área de automação da manufatura. In: 2o Congresso Brasileiro de Engenharia de Fabricação (COBEF), Uberlândia, MG, v. 18, 2003.

ANDÚJAR, J. M.; MEJÍAS, A.; MÁRQUEZ, M. A. Augmented Reality for the Improvement of Remote Laboratories: An Augmented Remote Laboratory. IEEE Transactions on Education, v. 54, n. 3, 2011.

BANGOR, A.; KORTUM, P.; MILLER, J. Determining what individual SUS scores mean: Adding an adjective rating scale. Journal of usability studies, Citeseer, v. 4, n. 3, p.114-123, 2009.

BERALDO, A. L. S.; OLIVEIRA, T.; STRINGINI, D. Laboratório remoto de FPGA com foco no ensino: Uma revisão sistemática da literatura. Revista Novas Tecnologias na Educação (RENOTE), v. 18, n. 1, 2020.

BRAGGIO, A. A. FPGA: processo de configuração com desenvolvimento de protótipo em placa de circuito impresso. Dissertação (B.S. thesis) - Universidade Tecnológica Federal do Paraná, 2014.

BROOKE, J. SUS: A "quick and dirty" usability. Usability Evaluation in Industry, v. 189, 1996.

LINDSAY, E. et al. Remote laboratories in engineering education: Trends in students'perceptions. In: AUSTRALASIAN ASSOCIATION FOR ENGINEERING EDUCATION. Proceedings of the 18th Conference of the Australasian Association for Engineering Education, 2007.

LOURENÇO, R. da S. Laboratórios remotos - um estudo para a PUC-Rio, 2014.

MEC. Diretrizes Curriculares Nacionais para os cursos de graduação na área da Computação, 2016.

NIELSEN, J. Usability 101: Introduction to usability. Disponível em: < https://www.nngroup.com/articles/usability-101-introduction-to-usability/ >. Acesso em: 21 maio 2021.

NUNNALLY, J. C. Psychometric theory (2nd edit.). New York, 1978.

PINTILIE, L. N. et al. Multifunctional socket for smart grid applications using ethernet over power lines and usb over ip technologies. In: IEEE International Conference on Applied and Theoretical Electricity (ICATE), 2018.

RASCHKE, C. A. The digital revolution and the coming of the postmodern university. Routledge, 2003.

RODRIGUEZ-GIL, L.; GÁRCIA-ZUBIA, J.; ORDUNA, P.; LOPES-DE-IPINA, D. Towards New Multiplatform Hybrid Online Laboratory Models. IEEE Transactions on Learning Technologies, vol. 10, no. 3, pp. 318-330, 2017.

SAURO, J.; LEWIS, J. R. Correlations among prototypical usability metrics: Evidence for the construct of usability. In: Proceedings of the SIGCHI conference on human factors in computing systems, 2009.

SILVA, T. A. V. et al. Sistema thin client para aplicações laboratoriais de ensino. Universidade Federal de Uberlândia, 2019.

VIDAL, N. F.; MENEZES, P. H. D. Laboratório real $\mathbf{x}$ laboratório virtual: possibilidades e limitações desses recursos no ensino de eletrodinâmica, 2015. 